\title{
AN ELECTRONIC TECHNIC FOR RECORDING THE MYODYNAMIC FORCES OF THE LIP, CHEEK, AND TONGUE
}

\author{
JAMES P. ALDERISIO, B.S., D.D.S., M.S., AND ROY LAHR, E.E.
}

University of Michigan, Ann Arbor, Mich.

$\mathrm{R}$

ECENTLY, at least five reports ${ }^{1-5}$ have been submitted and as many devices have been constructed to measure the forces acting on the dental arch. All of these have pertained to the measurement of occlusal biting forces. Feldstein ${ }^{6}$ employed the principle of hydraulics in a technic to measure the immediate forces on the buccal or lingual surface of a tooth.

The technic described herein has been proved sound in a laboratory study and, with refinements, is to be employed in a cross-sectional study of the University of Michigan High School and Elementary School children. It is capable of recording successively and accurately as many as seven pressures exerted by the lips, cheeks, and tongue upon various areas of the dental arch.

\section{PHYSICAL PRINCIPLES INVOLVED}

As the cross section of a carrying wire is decreased, its ability to carry an electrical current also is decreased, i.e., its resistance is increased. This principle has been utilized in a small filament of wire, the SR4 Baldwin Gauge, so that muscular pressure exerted on the gauge causes an elongation and changes the electrical conductivity of the wire.

Utilized in this capacity, a change in the force exerted produces a deformation of the gauge wire which can be plotted as a linear, straight line curve. Such a plotted curve indicates that one derives an output of voltage from the proper external measuring circuit which is proportional to the force applied. It then is possible to find the constant of proportionality at a point in the pressure range of the gauge, and this constant will maintain for any other point in the range. Any pressures outside the operating range will be so far from the linear constant that they will be easily detectable on the recorder. The gauges that were used registered a resistance within $0.3 \mathrm{ohm}$ of each other.

For the external circuit the principle of the balanced Wheatstone bridge was used with modifications. A Wheatstone bridge has four wire arms with three known values and permits the finding of the fourth. In this technic the ratio arms are one to one, since the standard and the unknown are both wire gauges and within $0.3 \mathrm{ohm}$ resistance of each other.

\footnotetext{
Prepared by the senior author in partial fulfllment of the requirements for a Master of Science degree in Orthodontics at the W. W. Kellogg Foundation Institute, University of Michigan.

Received for publication, Oct. 15, 1952 .
} 
In order to record successively the muscular forces acting on different areas of the mouth, the Wheatstone bridge which was used had been provided with this arrangement for switching between seven unknown gauges and a standard gauge. This arrangement was accomplished by the use of a scanning switch which included a motor to drive a cam wheel that activated the seven switches successively. Each switch had an unknown gauge balanced to the standard gauge. The balancing of each gauge was accomplished by a visual meter dial so that balancing was a quick, easy process. The balancer used was an Anderson Model 302 Bridge Balancer. In reality, therefore, there are seven Wheatstone bridges in one mounting, and all gauges are balanced to one gauge. A type of universal motor was used so that variation in the voltage to the motor secured a wide range of switching rates. Alternating current was used in the circuit because of the ease in amplifying the bridge voltage output. One thousand cycles per second current were used so that power line fields of sixty cycles per second would not interfere.

\section{PREPARATION OF GAUGE}

The gauges used are very sensitive and require protection as well as a definite means of insertion into the mouth. To meet this requirement a metal carrier, $2 \mathrm{~mm}$. thick, $4 \mathrm{~mm}$. wide, and $6 \mathrm{~mm}$. long, was constructed with a soldered base and eyelets. The eyelets served as a means for attaching a rubber separator which went between the teeth and which held the carrier in position in the mouth. In cases where spaces are present, due to absent teeth, the metal gauge carriers are ligated to the teeth. On each width, on its inside surface, a half-round tube was soldered. Into one of these tubes halfround shafting, which consisted of a flat stainless steel spring wire soldered on its top surface, was fitted. Hence, the shafting, when inserted into the half-round tube, made a modified cantilever bridge with the free end of the spring wire resting on the top surface of the opposite half-round tube. On the bottom surface of the flat spring wire, in the center, the gauge was glued. The center of the top surface of the flat spring wire carried the plunger which received the muscular pressure. The spring and sides of the carrier were sealed off with a loosely fitting thin rubber sheet. Through one side of the carrier passed the leads which connected with the collecting box. The induced electromotive force thus continued through the gauge balancer to the seanning switch and then on to the gauge indicator and recorder. To take a reading the gauges were placed in position in the mouth and allowed to warm to mouth temperature. The muscles over each gauge in turn were held away from the gauge while it was being balanced to the standard gauge. This balancing procedure was accomplished visually by zeroing the indicator needle of the balancer with the standard gauge. When all gauges were balanced to the standard gauge and no muscular pressure was in action, it was possible to measure the muscle tonus of the relaxed musculature in the area of the measuring gauge. When measurement of a muscle action then was recorded, the variation in the two readings measured the force exerted by that particular motion. 
The recording apparatus appropriate for this type of assembly was found to be that of the Brush Development Company which provided proportional readings of voltage as the applied pressure was increased. For the sample readings, a sound level recorder was used. The pressure on the gauge then became a logarithmic function of the balance point as recorded on the tape.

In the sample tracings that were made, two combinations of the gauges were used: one with five gauges on the labial and buccal surfaces, and a second group with four gauges on the labial and buccal surfaces and three on the lingual surfaces. The numbering of the gauges is shown in Fig. 1.

An analysis of the tracings indicates that the greater the depth of the recording, the smaller is the amount of pressure. The pointed dips in the tracings were due to the inertia of the recording stylus. Measurements for comparative studies and calibrations were made by measuring the length of the perpendicular as drawn in Fig. 2 for gauge No. 1.

The recordings in Fig. 2 were obtained while the patient was as relaxed as possible in order to secure the pressure of the oral musculature in a rest position. The patient's tongue was lying in the floor of the mouth away from the gauges on the lingual surfaces of the teeth. Under these conditions one obtains the long recording of the lingual gauges which signifies no pressure. At the same time the muscle pressure in the canine area of gauges 2 and 4 at rest, under the same conditions, produced a tracing similar to that of little or no pressure when compared to the recordings secured by tracings during the absence of lingual pressure. Gauge No. 1 showed a smaller recording than the others, signifying an increase of muscle force, and this increase in anterior gauge No. 3 was still greater as is shown by the greatly reduced tracing.

When the same individual was told to swallow, producing the subsequent increase of labial, buccal, and lingual pressure, Fig. 3 was secured. Gauge No. 1 recorded a greatly increased muscular force as revealed by the small amount of tracing in the second group of recordings. This increase exceeded that inerease in the anterior gauge No. 3 so that now the pressure on the molar gauge exceeded that on the anterior gauge. The lingual tracings, as recorded, reveal the lingual pressure exerted in swallowing when compared with those of Fig. 2. The second series of tracings of gauges 5, 6, and 7 showed the beginning of relaxation of the tongue's musculature and a corresponding elongation of recordings. Fig. 4 was the recording of the labial and buceal forces under three different conditions. The first group of five recordings was secured when no labial or buccal pressure was being exerted. This recording was accomplished by holding the cheeks and lips away from the gauges that were in the mouth. The recordings under the title "No Pressure" show the typical long record. Gauge No. 5 of this series did not have all of the cheek muscle off the gauge and hence produced a shorter tracing than the other four tracings in the first group. The second group of five recordings included those of the relaxed, "at rest" musculature. Here the situation repeats itself, as seen in Fig. 2, with little or no change in the 


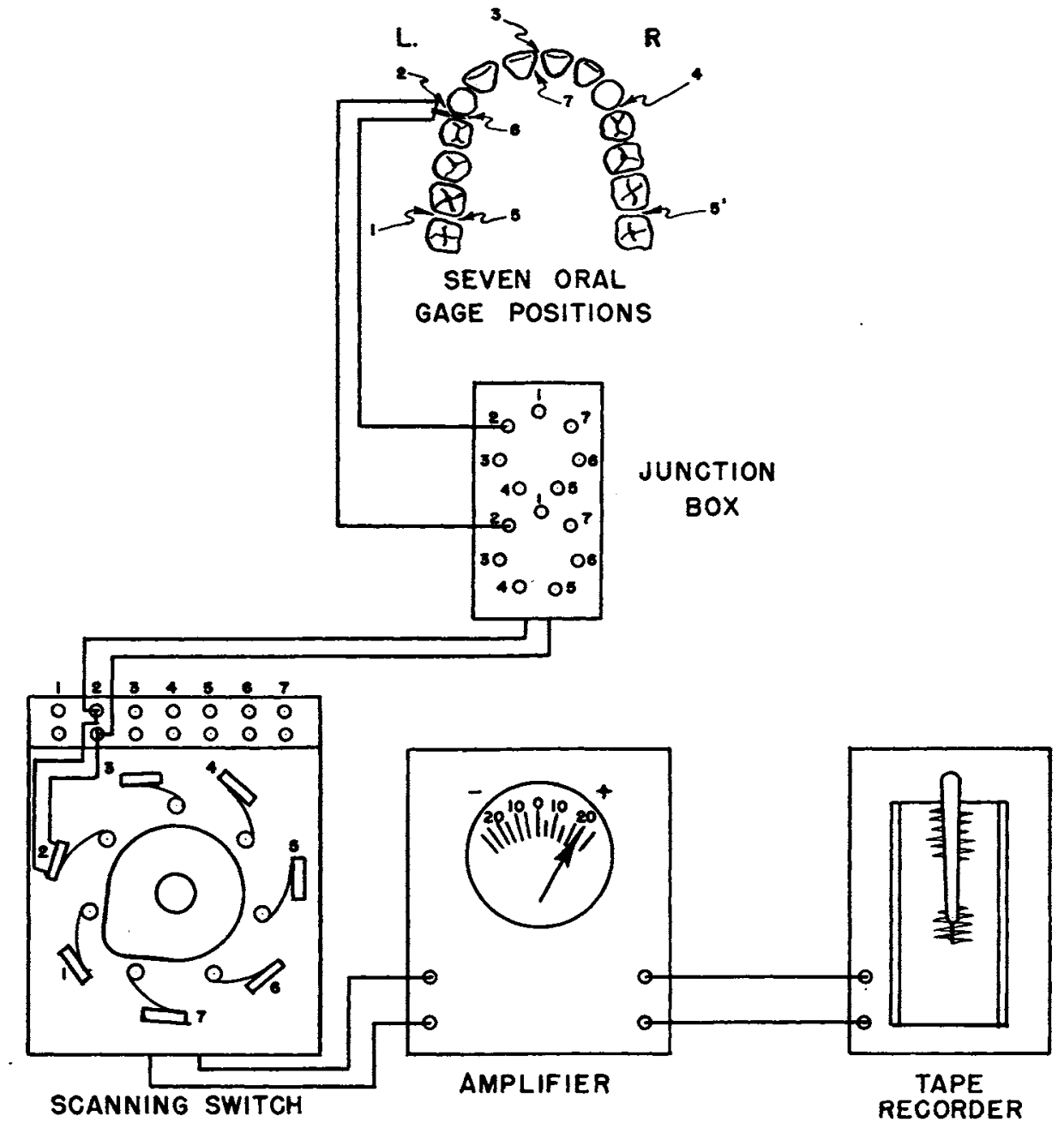

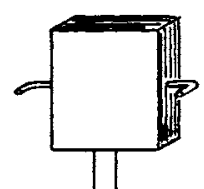

A

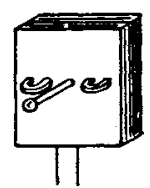

B

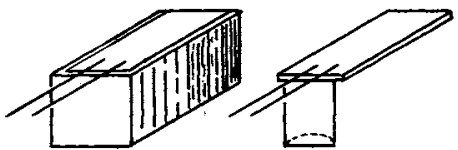

C

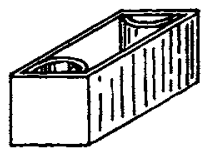

E

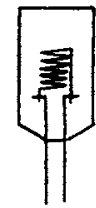

$\boldsymbol{F}$

\section{GAGE CARRIER DETAILS}

Fig. 1.-Electronic recording equipment arrangement. Top diagram shows positioning of the gauges in the mouth and connection to scanning switch with insulated cable by means of junction collecting box which has neck support for patient. Revolving cam wheel contacts individual G-RL micro switches which are connected to oral gauges and leads to the amplifier individual recorder.

Gauge Carrier Details:

A, Gauge carrier with loops for ligation.

$B$, Gauge carrier with eyelets and elastic which is held in place by self-curing acrylic.

$C$, Spring in position in gauge carrier.

$D$, Spring soldered to post.

$E$, Gauge carrier showing two half-round tubes, one to receive one-half round shafting and other as support of free end.

$F$, Theoretical figure of SR strain gauge. 
canine area, but with noticeable increase of force in the molar and incisor areas. The third group of recordings were those secured under the influence of muscle contraction during swallowing. Again the typical recording was determined of further increase in molar pressure exceeding that of the anterior increase, with the reaction of the cuspid area unchanged.

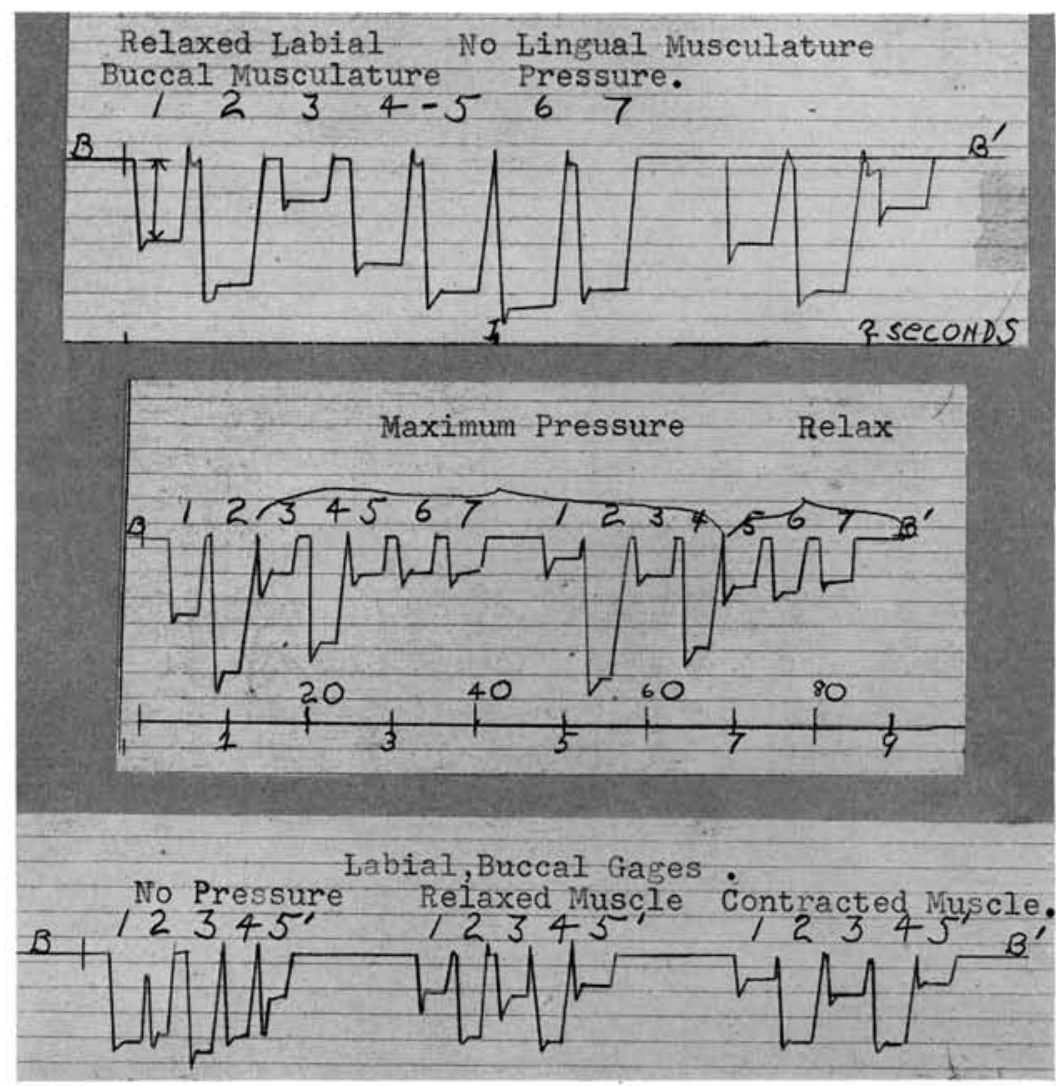

Fig. 2.

Fig. 3.

Figs. 2 to 4 .

From the tracings which have been presented as evidence of the ability of the myodynamic recorder to register successively the muscular force exerted on seven areas of the oral cavity, no attempt has been made to assign interpretative values to the tracings in this introductory study. Hesitancy to do so arose from the fear that such values might be misinterpreted as definitive values for the different areas. This report, therefore, merely presents a technic by which the myodynamic recorder can be utilized to make an accurate study of the oral forces.

\section{REFERENCES}

1. Howell, A. H., and Manly, R. S.: An Electronic Gauge for Measuring Oral Forces, $J$. D. Res. 27: 705-712, 1948.

2. Howell, A. H., and Brudevold, F.: Vertical Forces Used During Chewing of Food, J. D. Res. 29: 133-136, 1950 . 
3. Moyers, R. E.: Temporomandibular Muscle Contraction Patterns in Angle Class II, Division 1 Malocelusions: An Electromyographic Analysis, Am. J. Orthodontics 35: $837-855,1949$.

4. Muhlemann, H. R.: Periodontometry, a Method for Measuring Tooth Mobility, Oral Surg. 4: $1220-1233,1951$.

5. Neumann, H. H.: Measurement of the Effort Exerted in Chewing Various Foods, J. D. Res. 29: 463-468, 1950.

6. Feldstein, L.: An Instrument for Measuring Muscular Forces Acting on the Teeth, Am. J. Orthodontics 36: 856-859, 1950.

\section{Erratum}

In the article by Viktors Kalnins, "The Effect of X-Ray Irradiation Upon the Mandibles of Guinea Pigs Treated With Large and Small Doses of Ascorbic Acid," which appeared on page 177 of the April issue of the Journal, the second sentence in paragraph 3 read, "Furthermore, physiologic pharmacodynamic doses of vitamin $\mathrm{C}$, exceeding several times the dose of ascorbic acid, act as detoxicants." This sentenee should have been, "Furthermore, pharmacodynamic doses of Vitamin C exceeding several times physiologic dose of ascorbic acid, ..." 